Sinergias educativas

ISSN: 2661-6661

compasacademico@icloud.com

Grupo Compás

Ecuador

\title{
Desarrollo de la motricidad fina y gruesa en etapa infantil
}

Mendoza Morán, Angélica Mariela

Desarrollo de la motricidad fina y gruesa en etapa infantil

Sinergias educativas, vol. 2, núm. 2, 2017

Grupo Compás, Ecuador

Disponible en: http://www.redalyc.org/articulo.oa?id=573561679005

Esta obra está bajo una Licencia Creative Commons Atribución-NoComercial-SinDerivar 4.0 Internacional. 


\title{
Desarrollo de la motricidad fina y gruesa en etapa infantil
}

\author{
Development of fine and gross motor skills in children stage \\ Angélica Mariela Mendoza Morán \\ angelicamendoza@gmail.com \\ Profesora Unidad Educativa Nueva España, Ecuador \\ (iD http://orcid.org/0000-0003-3946-1384
}

Sinergias educativas, vol. 2, núm. 2, 2017

Grupo Compás, Ecuador

Recepción: 15 Marzo 2017 Aprobación: 25 Noviembre 2017

Redalyc: http://www.redalyc.org/ articulo.oa?id $=573561679005$

\section{BY-NC-ND}

Resumen: El arti\#culo parte de la experiencia de varios docentes al observar que estudiantes de 1 a 6 an\#os presentan una incapacidad motriz. Con el objetivo de descubrir la importancia que tiene la motricidad en el proceso de ensen\#anza y aprendizaje en los estudiantes en el salo\#n; en esta investigacio\#n se describen claramente los tipos de motricidad que se deben desarrollar en la etapa infantil. La metodologi\#a considera para el estudio de este arti\#culo es la etnografi\#a reflexiva y el caso cualitativo. Se obtiene como resultado que la motricidad se complementa con elementos materiales del aula para ensen\#ar y que las ane\#cdotas son una opcio\#n motriz en el aprendizaje. Como conclusio\#n es que mediante estos ejercicios o actividades llamamos la atencio\#n del personal docente que trabaja con esta edad infantil para que lo utilicen en vi\#as de mejorar las tareas a desarrollar con los educandos.

Palabras clave: Educacio\#n fi\#sica, psicomotricidad, motricidad fina .

Abstract: The article is based on the experience of several teachers when observing that students from 1 to 6 years have a motor disability. With the aim of discovering the importance of motor skills in the teaching and learning process in students in the classroom; This research clearly describes the types of motor skills that should be developed in the childhood stage. The methodology considered for the study of this article is the reflective ethnography and the qualitative case. It is obtained as a result that motor skills are complemented with material elements of the classroom to teach and that anecdotes are a driving option in learning. In conclusion, through these exercises or activities, we call the attention of the teaching staff who work with this child age to be used to improve the tasks to be developed with the students

Keywords: Physical education, psychomotor skills, fine motor skills.

\section{INTRODUCCIÓN}

El desarrollo motriz es de vital importancia ya que se presentan distintas etapas de movimientos esponta\#neos y descontrolados en los nin\#os y nin\#as.

La psicomotricidad, divide la motricidad del nin\#o en: fina y gruesa. La motricidad fina se refiere a las acciones que implican pequen\#os grupos musculares de cara, manos y pies, concretamente, a las palmas de las manos, los ojos, dedos y mu\#sculos que rodean la boca. Es la coordinacio\#n entre lo que el ojo ve y las manos tocan (o\#culo-manual).

La motricidad gruesa es aquella que hace referencia a todas las acciones que implican grandes grupos musculares, en general, se trata de movimientos grandes del cuerpo del nin\#o o de todo el cuerpo.

Existen estudiantes que presentan una imposibilidad motriz. Segu\#n la estadi\#stica del INEGI y experiencias de docentes dentro del salo\#n 
El aprendizaje de la escritura es un proceso evolutivo que se desarrolla gradualmente. Los nin\#os y nin\#as no esta\#n preparados para la escritura en forma homoge\#nea cuando entran a primer an\#o escolar, tampoco progresan todos a un mismo ritmo en su aprendizaje. La ensen\#anza de la lectura y la escritura no siempre va a la par. Muchos nin\#os saben leer y sin embargo tienen dificultades para escribir.

Actualmente esta\# comprobado que en los primeros an\#os es recomendable las clases y actividades fi\#sicas ya que enriquecen la vida de los nin\#os, el desarrollo fi\#sico, social y cognitivo. En ninguna otra etapa de la vida es tan importante la educacio\#n fïsica como en los an\#os preescolares.

La psicomotricidad ocupa un lugar importante en la educacio\#n infantil, ya que esta\# totalmente demostrado que en la primera infancia existe una gran interdependencia en los desarrollos motores, afectivos e intelectuales.

La teori\#a de Piaget afirma que la inteligencia se construye a partir de la actividad motriz del nin\#o o nin\#a. Desde la psiquiatri\#a infantil destaca el papel de la funcio\#n to\#nica, entendiendo que no es so\#lo la tela de fondo de la accio\#n corporal sino un modo de relacio\#n con el otro.

Por tanto en los primeros an\#os de la educacio\#n del nin\#o o nin\#a, hasta los siete an\#os aproximadamente, toda la

educacio\#n es psicomotriz porque todo el conocimiento, el aprendizaje, parte de la propia accio\#n del estudiante sobre el medio y las experiencias ya que son manifestaciones diferentes aunque interdependientes de un ser u\#nico: EL NIN\#O/A.

La motricidad es el dominio que el ser humano es capaz de ejercer sobre su propio cuerpo. Es algo integral ya que intervienen todos los sistemas de nuestro cuerpo. Va ma\#s alla\# de la simple reproduccio\#n de movimientos y gestos, involucra la espontaneidad, la creatividad, la intuicio\#n, etc., tiene que ver con la manifestacio\#n de intencionalidades y personalidades.

La motricidad tambie\#n manifiesta todos los movimiento del ser humanos. Estos movimientos determinan el comportamiento motor de los nin\#os/ as de 0 a 6 an\#os que se manifiesta por medio de habilidades motrices ba\#sicas, que expresan a su vez los movimientos naturaleza del hombre. (Gonza\#lez, 2001)

En la siguiente figura se explica claramente los tipos de motricidad que existen:

La motricidad fina La motricidad fina implica el control voluntario y preciso de los movimientos de la mano y los dedos. Es una habilidad fundamental para realizar una gran cantidad de actividades escolares. Es habitual que al comienzo de la Educacio\#n Infantil existan nin\#os y nin\#as que tengan dificultades en el dominio de esta habilidad. A continuacio\#n se detallan algunas actividades para desarrollar la motricidad fina: Actividades con plastilina: pellizcar trocitos de plastilina, hacer bolitas de pequen\#o taman\#o, aplastar las bolitas con los dedos; extender plastilina sobre alguna superficie lisa; realizar juego libre con la plastilina. Actividades con papel: rasgar papel (utilizando los dedos 
pulgar e i\#ndice); hacer bolitas arrugando el papel, doblar papel. Dibujar libremente: garabateo, darles un tema concreto. Pintura de dedos: pintar libremente o seguir caminos sen\#alados y colorear. Ensartar pasta (macarrones): con lana y hacer collares. Jugar con pinzas de la ropa: colocarlas en la ropa y en cartulina. Abrochar y desabrochar cremalleras, botones. Abrir y cerrar envases. Formar torres con bloques Picar li\#\#neas y siluetas con el punzo\#n. Estirar gomas ela\#sticas, globos... Ejercicios de imitacio\#n y estimulacio\#n con dedos y manos. Actividades para desarrollar la destreza de las manos.

Tocar palmas, primero libremente, despue\#s siguiendo un ritmo.

Llevar uno o ma\#s objetos en equilibrio en la palma de la mano, primero en una mano, despue\#s en las dos.

Hacer trazos libremente sobre la arena y/o sobre el agua.

Realizar gestos con las manos acompan\# ando a canciones infantiles, un juego divertido desde que son bebe\#s.

Girar las manos, primero con los pun\#os cerrados, despue\#s con los dedos extendidos.

Mover las dos manos simulta\#neamente en varias direcciones (hacia arriba, hacia abajo, movimiento circular)

Imitar con las manos movimientos de animales (leo\#n moviendo las garras, pa\#jaro volando...) o de objetos (aspas del molino, he\#lices de helico\#ptero.

Abrir una mano mientras se cierra la otra, primero despacio, luego ma\#s ra\#pido.

Actividades para desarrollar la destreza de los dedos.

Abrir y cerrar los dedos de la mano, primero simulta\# neamente, luego alterna\#ndolas. Ir aumentando la velocidad.

Juntar y separar los dedos, primero libremente, luego siguiendo o\#rdenes.

Tocar cada dedo con el pulgar de la mano correspondiente, aumentando la velocidad.

Con la mano cerrada, sacar los dedos uno detra\#s de otro, empezando por el men\#ique.

Con las dos manos sobre la mesa levantar los dedos uno detra\#s de otro, empezando por los men\#iques.

"La motricidad fina es la accio\#n de pequen\#os grupos musculares de la cara y los pies. Movimientos precisos de las manos, cara y los pies”. (Belkis, 2007)

Los nin\#os deben comenzar a desarrollar su motricidad fina desde muy pequen\#os, siendo capaces muchas veces de ir reforzando lo que ma\#s les va costando o simplemente ir pidiendo ayuda para que lo orienten. La caligrafi\#a es una de las te\#cnicas en las cuales se va desarrollando la motricidad fina en los estudiantes.

Coordinacio\#n fone\#tica.

Es un aspecto dentro de la motricidad muy importante a estimular y a seguir de cerca para garantizar un buen dominio de la misma. 
El nin\#o en los primeros meses de vida descubre las posibilidades de emitir sonidos sin embargo no tiene la madurez necesaria que le permita una emisio\#n sistema\#tica de cualquier sonido.

El nin\#o ha iniciado el aprendizaje que le permitira\# llegar a la emisio\#n correcta de palabras.

Poco a poco ira emitiendo si\#labas y palabras que tendra\#n igualmente una respuesta, especialmente cuando no se trate de una conversacio\#n sino de un juego de decir cosas y aprender nuevas palabras, hacer sonidos de animales u objetos. Apartirde1a2an\#osdeedadel nin\#o tiene la madurez para iniciar un lenguaje, no contendra\# demasiadas palabras, las frases sera\#n simples y en el mejor de los casos podra\# hacerlo ra\#pidamente.

Estos juegos motrices tendra\#n que continuar sobre todo para que el nin\#o vaya adquiriendo un nivel de conciencia ma\#s elevado.

Entre los 2 y 3 an\#os de edad el nin\#o tiene las posibilidades para sistematizar su lenguaje, para perfeccionar la emisio\#n de sonidos y para concienciar la estructuracio\#n de las frases y hacerlas cada vez ma\#s complejas.

Al final del tercer an\#o quedara\#n algunos sonidos para perfeccionar y unas irregularidades gramaticales y sinta\#cticas a consolidar.

Todo el proceso de consolidacio\#n ba\#sica se realizara\# entre los tres y cuatro an\#os, cuando el nin\#o puede y tendra\# que hablar con una perfecta emisio\#n de sonidos y por consiguiente con un verdadero dominio del aparato fonador.

El resto del proceso de maduracio\#n lingu\#i\#stica y de estilo se hara\# a la larga en el transcurso de la escolarizacio\#n y la maduracio\#n del nin\#o.

Motricidad gruesa Para trabajar la motricidad gruesa es

importante realizar actividades que estimulen el cuerpo. Estas acciones son las que llevan a los nin\#os o nin\#as a cambiar la posicio\#n de su cuerpo y ayudan a controlar su fuerza.

La motricidad gruesa es la habilidad que el nin\#o va adquiriendo para mover armoniosamente los mu\#sculos del cuerpo de modo que puede, poco a poco, mantener el equilibrio de la cabeza, del tronco y extremidades para sentarse, gatear, ponerse de pie y desplazarse con facilidad, caminando o corriendo (Belkis, 2007)

A continuacio\#n se detallas algunas actividades para desarrollar la motricidad gruesa: Derribar pira\#mides de objetos. Para este juego puedes usar latas vaci\#as que ubicara\#s en forma de pira\#mide. Necesitara\#s tambie\#n de una pelota, no muy pesada y poner al nin\#o del objetivo. Ahora debe tirar la pelota buscando apuntar y derrumbar las latas. Cuando practicamos este juego se controla la fuerza y la coordinacio\#n de movimientos del cuerpo, lo cual es esencial manejar antes de comenzar a escribir.

Pasar objetos de diversos taman\#os y pesos para que agarre: puedes usar elementos que sean de dimensiones diferentes y un poco pesadas, sin ser excesivo, para pasar a los nin\#os. Estos deben agarrarlos y de esta forma trabajara\#n su resistencia y fuerza. Puedes mejorar la actividad haciendo que el infante arroje estos objetos a lo lejos. 
Atrapar la pelota o el globo: dependiendo de la fuerza del nin\#o pueden jugar a lanzar un globo o una pelota no muy

pesada. El pequen\#o debera\# intentar agarrarla y tambie\#n debe arrojarla hacia ti. Para comenzar puedes usar un globo, ya que es un objeto liviano que no lo lastimara\# y tambie\#n tiene un movimiento lento que le permite reaccionar a tiempo.

Jugar en el parque: esta alternativa integra en realidad muchos juegos $y$ es que los parques infantiles ofrecen una amplia variedad de actividades para que los nin\#os realicen. Alli\# pueden correr, saltar la cuerda, pasar por las barras, entre otras actividades que incentivan el equilibrio y permiten trabajar la psicomotricidad gruesa. Cruzar obsta\#culos: se pueden colocar diferentes objetos, como bancos o maletas, para que el estudiante los cruce de un lado a otro. Tambie\#n puedes hacer uso de una cuerda templada para hacerlo saltar y de esta forma trabajar su motricidad.

Armar rompecabezas del cuerpo humano. Si el maestro no cuenta con este material, puede recortar de revistas figuras humanas completas y descomponer en 6, 8 y 10 partes para que el nin\#o arme y pegue sobre una cartulina.

Autores de gran prestigio, han realizado caracterizaciones motrices en nin\#os de diferentes edades. Bryant J. Cratty bio\#logo de los Estados Unidos, basado en la aplicacio\#n de una lista de control describe las caracteri\#sticas del comportamiento perceptivo motor de los nin\#os(as), las teori\#as evolutivas de los psico\#logos J. Piaget y H. Wallon, citado por Aquino y Zapata, nos permiten apreciar los logros motores de sujetos estudiados, asi\# como las referencias sobre el desarrollo motor y los movimientos rectores de la pedagoga alemana Kahte Lewin, brindan una gran informacio\#n al respecto.

La investigacio\#n de este arti\#culo se lo relaciono\# primero con el estudio del caso cualitativo, que esta\# centrado en el conocimiento holi\#stico de un feno\#meno contempora\# neo dentro de su contexto real, en el que los li\#mites entre el feno\#meno y su contexto no son claros y exigen mu\#ltiples fuentes de informacio\#n.

De acuerdo con esta definicio\#n, esta estrategia metodolo\#gica permite la contextualizacio\#n y la particularizacio\#n del caso, aunque no la generalizacio\#n de datos. Tiene como finalidad descubrir y analizar situaciones u\#nicas, analizar las relaciones en que tiene lugar el caso objeto de estudio y desarrollar la capacidad de observacio\#n de la realidad.

El estudio de caso es un me\#todo que favorece la reflexio\#n sobre la pra\#ctica y facilita la comprensio\#n. (Stake, 1999) El otro tipo de investigacio\#n que aporto\# al estudio es la etnografi\#a reflexiva. "Es una descripcio\#n e interpretacio\#n de un grupo social o sistema cultural donde el investigador estudia la forma de vida, costumbres, conductas y observa aprendizajes de las comunidades". (Norman Denzin, Yvonna Lincoln, 2012).

La cual se pretende descubrir las emergencias de los sujetos en cualquier a\#mbito de su vida. Por tal razo\#n, la etnografi\#a reflexiva es de gran relevancia en la pretensio\#n que 
este estudio tuvo por los sentidos que los maestros le otorgan a la motricidad como un saber para la ensen\#anza. La poblacio\#n considerada para el estudio del arti\#culo fueron docentes y estudiantes de la unidad educativa. La cual constituyo\# en que los docentes encargados en especial la maestra de cultura fi\#sica deben observar la incapacidad motriz de los estudiantes sea esta fina o gruesa y realizar las diferentes actividades expuestos en este arti\#culo. El actuar del docente acompan\#ado de la motricidad permitira\# comprender al estudiante y la ensen\#anza de otra forma.

Existe una amplia descripcio\#n de las peculiaridades de la motricidad en cada grupo de nin\#os en la etapa preescolar. Esta informacio\#n puede ser de utilidad como referencia de uno de los resultados a los que se pueden alcanzar en el estudio de la motricidad infantil.

\section{CONCLUSIONES}

Con la realizacio\#n del arti\#culo se busca enriquecer, desarrollar y estimular al estudiante dentro de la motricidad y su conexio\#n con los procesos de aula.

El trabajo con la poblacio\#n para desarrollar la motricidad en infantes, fue muy enriquecedor tanto para los educadores como los educandos dejando grandes aprendizajes que contribuyeron a nuestro proceso de formacio\#n y al proceso de formacio\#n de los estudiantes.

Los enfoques y las actividades que

se sugieren en esta investigacio\#n esta\#n fuertemente relacionados con el enriquecimiento de la ensen\#anza y aprendizaje de los nin\#os en la escuela. Le ayuda a relacionarse con los dema\#s, con el entorno y el desarrollo de los sentidos.

En la exploracio\#n de la importancia motriz para ensen\#ar a los maestros, se visibiliza en un juego permanente de ir y venir respecto a las diferentes experiencias acade\#micas, personales y familiares que tiene el docente, lo que permite manejar un estilo especial y particular para ensen\#ar.

La manifestacio\#n de la motricidad en sus alternativas instrumentales, co\#sicas o fenomenales se percibe como un elemento facilitador en la ensen\#anza, en los procesos cognitivos y de motricidad que deben apoderarse del espacio acade\# mico.

Cuando esta tiene lugar con el objetivo de alcanzar comprensio\#n y entendimiento del tema y de si\# mismo, se hace imprescindible una explicacio\#n, exposicio\#n y comunicacio\#n clara del docente, quien debera\# integrar su motricidad y la de sus estudiantes en estos procesos acade\#micos; pero, adema\#s, obligar a conjugar actividades que le den significado y sentido a lo realizado en este contexto.

Son muchas las realidades de la relacio\#n motricidad-mente que se manifiestan en el aula; estas constituyen una alternativa de estudio y desarrollo que puede vincularse en los saberes de orden pedagógico que pueden y deben tener los maestros

orden pedago\#gico que pueden y deben tener los maestros. 
Los aportes de la motricidad en la ensen\#anza dejan abierto otro camino para seguir viviendo y construyendo la formacio\#n de docentes y los suen\# os de una sociedad mejor.

\section{Referencias}

Belkis, P. H. (2007). LA MORTICIDAD FINA EN LA ETAPA INFANTIL. Cuba: portal educativo.

Sánchez, A. (26 de enero de 2015). Motricidad Gruesa: Actividades y ejercicios esenciales para trabajar. España: Educapeques. Recuperado de http://www. educapeques.com/escuela-de-padres/ motricidad-gruesaactividades.html

Franco Jime\#nez, A.M., \& Ayala Zuluaga, J.E. (2011). Aportes de la motricidad en la ensen\#anza. Revista Latinoamericana de Estudios Educativos. 7(2), 95-119.

Gonza\#lez, C. (2001). Educacio\#n Fi\#sica en el preescolar. Cuba: Deportes de cuba.

Norman Denzin, Yvonna Lincoln. (2012). Manual de investigacio\#n cualitativa. Espan\#a: Gedisa.

Stake, R. (1999). Investigacio\#n con estudio de casos. Madrid: Ediciones Morata. 